\title{
Small groups of finite Morley rank with involutions
}

\author{
Adrien Deloro*and Eric Jaligot ${ }^{\dagger}$
}

June 21, 2008

\begin{abstract}
By analogy with Thompson's classification of nonsolvable finite $\mathrm{N}$ groups, we classify groups of finite Morley rank with solvable local subgroups of even and of mixed type. We also consider miscellaneous aspects concerning "small" groups of finite Morley rank of odd type.
\end{abstract}

\section{Introduction}

When they are present, involutions play a major role in the classification of infinite simple groups of finite Morley in a way much reminiscent of their use in the Classification of the Finite Simple Groups. This is at least the goal of Borovik's Program to tranfer as much as possible of arguments based on involutions from the finite case to the case of finite Morley rank. In both cases, most critical configurations occur when considering "small" groups, which are at the same time, and by far, the most difficult to handle. In the present paper we will consider the easiest cases among these remarkably difficult cases.

Groups of finite Morley rank are equipped with a rudimentary notion of dimension on their first-order definable sets which behaves, or at least can be seen as, an abstract version of the Zariski dimension of algebraic varieties over algebraically closed fields. We refer to [BN94, $\mathrm{ABC} 08$ ] for the developments of the theory of groups of finite Morley rank and its links with finite group theory and algebraic group theory, which it encapsulates in a much more general and unified theory. Finite groups are exactly the groups of Morley rank 0, and for algebraic groups over algebraically closed fields (with no additional structure) the Morley rank corresponds to the geometric Zariski dimension.

It is known from [BBC07] that a connected group of finite Morley has either trivial or infinite Sylow 2-subgroups. Considering connected groups of finite Morley rank with involutions one has thus only to focus on groups with infinite Sylow 2-subgroups. Groups without involutions lead to situations similar to

\footnotetext{
*Rutgers University

${ }^{\dagger}$ Corresponding author - Université de Lyon, CNRS and Université Lyon 1
} 
that of the Feit-Thompson (Odd Order) Theorem in finite group theory, with no known infinite analog and actually different problems in this case Jal01a.

The preliminary result for Borovik's Program in presence of involutions is the following.

Fact 1 [BP90] Let $G$ be a group of finite Morley rank. Then Sylow 2-subgroups of $G$ are conjugate and if $S$ is one of them, then $S^{\circ}$ is nilpotent and a central product, with finite intersection, of a 2-torus and a 2-unipotent subgroup.

As usual, Sylow p-subgroups are defined as the maximal $p$-subgroups. A 2-torus is a divisible abelian 2-group of finite Prüfer rank, and a 2-unipotent group is a definable connected (nilpotent) 2-group of bounded exponent. These are similar in nature to the Sylow 2-subgroup structure of an algebraic group over an algebraically closed field of characteristic different from and equal to 2 , respectively. Accordingly, we usually say that the group $G$ has the follwing type, depending on the nontriviality of the 2 -torus $T$ and/or of the 2-unipotent subgroup $U$ :

\begin{tabular}{c|cc} 
& $U \neq 1$ & $U=1$ \\
\hline$T \neq 1$ & Mixed & Odd \\
$T=1$ & Even & Degenerate
\end{tabular}

We note that the terminology "Degenerate" has been adopted at a time when connected groups of finite Morley rank with finite but nontrivial Sylow 2-subgroups were possibilities not entirely excluded, but since [BBC07] it seems more informative to speak just of groups without involutions as far as connected groups are concerned.

For some reasons related to a global unipotence theory in groups of finite Morley rank explained in [Jal08b, [FJ08, the following notation may be used. For a group $G$ of finite Morley rank, $\pi$ a set of primes and $p$ a prime, we let $U_{(\infty, 0), \pi}(G)$ denote the subgroup of $G$ generated by definable decent tori of $G$ which coincide with the definable hull of their Hall $\pi$-subgroups, and $U_{(p, \infty)}(G)$ the subgroup of $G$ generated by its $p$-unipotent subgroups. In general, a decent torus is a definable divisible abelian group of finite Morley rank which coincides with the definable hull, that is the smallest definable containing subgroup [ABC08, §I 2.3], of its (divisible) torsion subgroup, and for an arbitrary prime $p$ a $p$-unipotent group is a definable connected nilpotent $p$-subgroup of bounded exponent. Subgroups of the form $U_{(\infty, 0), \pi}(G)$ and $U_{(p, \infty)}(G)$, and more generally any subgroup generated by an arbitrary family of definable connected subgroups, are definable and connected by a well known application of Zilber's generation lemma [BN94, §5.4].

When considering only the prime 2 , this allows one to define naturally the odd and even parts of an arbitrary group of finite Morley rank. In particular, and as the letters "T" and "U" are reserved for "torus" and "unipotent" respectively, one uses as in $\mathrm{ABC} 08$ the following simpler notation for the odd and even parts of a group $G$ of finite Morley rank: 


$$
T_{2}(G)=U_{(\infty, 0),\{2\}}(G) \text { and } U_{2}(G)=U_{(2, \infty)}(G) .
$$

Any group $G$ of finite Morley rank has a solvable radical, that is a unique maximal definable solvable normal subgroup, usually denoted $R(G)$ BN94, Theorem 7.3]. Following the general classification of simple groups of finite Morley rank of even type as algebraic groups over algebraically closed fields $\mathrm{ABC} 08$, the even part of an arbitrary group of finite Morley rank is best described as follows.

Fact 2 [ABC08, Proposition II 4.8 and Proposition X 1] Let $G$ be a group of finite Morley rank. Then $U_{2}(G)$, modulo its solvable radical, is a direct product of finitely many definable simple algebraic factors over algebraically closed fields of characteristic 2 .

The most general notion of "smallness" for a group of finite Morley rank, incorporating notably all solvable groups and Chevalley groups of type $\mathrm{PSL}_{2}$ and $\mathrm{SL}_{2}$ over algebraically closed fields, is the following.

Definition 3 A group of finite Morley rank is locally ${ }^{\circ}$ solvable $^{\circ}$ if the connected component of the normalizer of any infinite solvable subgroup is solvable. This is equivalent to require that the connected component of the normalizer of any nontrivial definable connected abelian is solvable by [DJ07a, Lemma 3.4 (4)].

We refer to DJ07a for a detailed study of such groups without any special assumption on the presence of involutions, and their analogies with those encountered in the Feit-Thompson Theorem and in Thompson's classification of finite " $N$-groups" with involutions Tho68, Tho70, Tho71, Tho73.

We note that the latter series of papers also corresponds in the finite case to a transfer from simplicity to nonsolvability of certain arguments for "small" groups, and one of the goals of the present paper concerning groups of finite Morley rank is the same. In particular, we will study nonsolvable locally ${ }^{\circ}$ solvable $^{\circ}$ groups of even and mixed types in Sections 1.1 and 1.2 respectively, by using results and/or technics from the study of simple groups of finite Morley rank ABC08. As naturally expectable, at least according to a long-standing feeling that groups of finite Morley rank resemble algebraic groups, our conclusion will be the following.

Theorem 4 Let $G$ be a locally solvable group of finite Morley rank with an infinite Sylow 2-subgroup. Then exactly one of the following three cases occur.

(1) $G^{\circ}$ is solvable.

(2) $G^{\circ} \simeq \mathrm{PSL}_{2}(K)$ for some algebraically closed field $K$ of characteristic 2, in which case $G=G^{\circ} \times E$ fome some finite subgroup $E$.

(3) $G^{\circ}$ is nonsolvable and has odd type. 
The case of nonsolvable connected groups of odd type has been studied in the thick series of consecutive works [Jal00, CJ04, BCJ07, Del07b, Del07a, Del08b, more precisely in the simple case, implying in this process large portions of the current developments of the theory of groups of finite Morley rank. A kind of "final" version of this voluminous work will be found in DJ07b. As a preparation, we will consider here certain specialized topics concerning groups of odd type, the case of solvable groups of odd type in Section 1.4 with generalities on involutive actions in Section 1.3, and the case of groups of odd type with "very small" Sylow 2-subgroups in Section 1.5.

In Section 1.6 we will also consider centralizers of involutions in groups of finite Morley rank when such involutions satisfy certain geometric properties reminiscent of small groups such as $\mathrm{PSL}_{2}$. This requires specific arguments analogous to the Cartan polar decomposition.

\subsection{Locally $^{\circ}$ solvable $^{\circ}$ groups of even type}

As in the case of simple groups $\mathrm{ABC} 08$, the elimination of connected nonsolvable groups of mixed type primarily depends on a classification of even type groups in the context of locally ${ }^{\circ}$ solvable $^{\circ}$ groups. The most relevant statement is the following.

Theorem 5 Let $G$ be a locally solvable group of finite Morley rank of even type. Then exactly one of the following two cases occur.

(1) $G^{\circ}$ is solvable, or

(2) $G^{\circ} \simeq \mathrm{PSL}_{2}(K)$ for some algebraically closed field $K$ of characteristic 2, in which case $G=G^{\circ} \times E$ fome some finite subgroup $E$.

We proceed to the proof of Theorem 5 . Let $G$ be a locally ${ }^{\circ}$ solvable $^{\circ}$ group of finite Morley rank of even type, which may be assumed to be connected as long as one considers only its connected component. We then have

$$
R\left(U_{2}(G)\right) \unlhd U_{2}(G) \unlhd G .
$$

If $R^{\circ}\left(U_{2}(G)\right)$ is nontrivial, then $U_{2}(G)$ is solvable by local ${ }^{\circ}$ solvability ${ }^{\circ}$ of $G$. It follows then that $G^{\circ}$ is solvable by local $^{\circ}$ solvability ${ }^{\circ}$ again, so we are in case (1) of Theorem 5 .

Assuming now that we are not in case (1) of Theorem 5, we have thus $R\left(U_{2}(G)\right)$ finite. Dividing $U_{2}(G)$ by its finite solvable radical, one gets a semisimple group, which is still locally ${ }^{\circ}$ solvable $^{\circ}$ by [DJ07a, Lemma 3.5]. Let

$$
H=U_{2}(G) / R\left(U_{2}(G)\right) .
$$

By Fact 2, $H$ is a direct product of finitely many definable normal simple subgroups. One sees that it is in fact a single definable normal simple subgroup by local $^{\circ}$ solvability ${ }^{\circ}$ of $H$. Hence $H$ is a simple group of even type. 
By the classification of the simple groups of even type, the main theorem of $\mathrm{ABC} 08, H$ is a simple algebraic group over an algebraically closed field of characteristic 2 . By local ${ }^{\circ}$ solvability ${ }^{\circ}$ of $H$, one concludes that

$$
H \simeq \mathrm{PSL}_{2}(K)
$$

for some algebraically closed field $K$ of characteristic 2 . The analysis can be continued as follows.

Lemma $6 Z\left(U_{2}(G)\right)=1$.

Proof. We have $Z\left(U_{2}(G)\right) \leq R\left(U_{2}(G)\right)$ which is finite, and in fact one has equality by [DJ07a, Fact 3.14].

Now $U_{2}(G)$ is a connected group. Its commutator subgroup is definable and connected by a well known corollary of Zilber's generation lemma BN94, Corollary 5.29]. As $U_{2}(G) / Z\left(U_{2}(G)\right)=H$ is a nonabelian simple connected group, one gets that $U_{2}(G)^{\prime}$ covers $H$, and thus $U_{2}(G)=Z\left(U_{2}(G)\right) \cdot U_{2}(G)^{\prime}$. Now finiteness of the center together with the connectedness of $U_{2}(G)$ forces that $U_{2}(G)$ is perfect, that is equal to its commutator subgroup.

Then the result of [AC99] on central extensions of algebraic groups implies that $U_{2}(G)$ is a Chevalley group over the same field $K$. As we are in characteristic 2 and dealing with $\mathrm{PSL}_{2}$, we conclude that the center is trivial.

At this point we have $U_{2}(G) \simeq \mathrm{PSL}_{2}(K)$ for some algebraically closed field $K$ of characteristic 2 .

Lemma $7 G=U_{2}(G)$.

Proof. Let $U$ be a maximal 2-unipotent subgroup of $U_{2}(G)$. By conjugacy of such subgroups in $U_{2}(G) \simeq \mathrm{PSL}_{2}$, a Frattini Argument gives

$$
G=U_{2}(G) \cdot N^{\circ}(U)
$$

and the factor $N^{\circ}(U)$ is solvable by local ${ }^{\circ}$ solvability ${ }^{\circ}$. Now $N^{\circ}(U)$ acts on $U_{2}(G) \simeq \mathrm{PSL}_{2}(K)$ by inner automorphisms as $\mathrm{PSL}_{2}$ has no graph automorphisms. In particular

$$
N^{\circ}(U)=N_{U_{2}(G)}(U) \times C_{G}\left(U_{2}(G)\right)
$$

and as the latter factor is finite by local $^{\circ}$ solvability ${ }^{\circ}$ of $G$ and nonsolvability of $U_{2}(G) \simeq \mathrm{PSL}_{2}$, one even has by connectedness of $N^{\circ}(U)$ that $N^{\circ}(U)=$ $N_{U_{2}(G)}(U) \leq U_{2}(G)$, and it follows that $G=U_{2}(G) \cdot N^{\circ}(U) \leq U_{2}(G)$.

We have thus shown that $G \simeq \mathrm{PSL}_{2}(K)$ for some algebraically closed field $K$ of characteristic 2 whenever $G$ is connected and not solvable as in case (1) of Theorem 5 To complete the statement as in case (2) of that theorem, it just remains to show the following. 


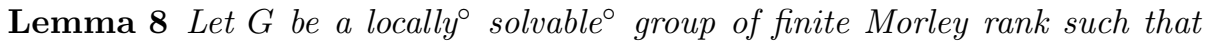
$G^{\circ} \simeq \mathrm{PSL}_{2}(K)$ for some algebraically closed field $K$ of characteristic 2 . Then $G=G^{\circ} \times E$ fome some finite subgroup $E$.

Proof. Again, by a Frattini Argument, $G=G^{\circ} \cdot N(U)$ for some maximal 2-unipotent subgroup $U$ of $G^{\circ}$, and as there are no graph automorphisms of $\mathrm{PSL}_{2}$ we get $N(U)=N_{G^{\circ}}(U) \times C_{N(U)}\left(G^{\circ}\right)$, and thus $G=G^{\circ} \times C_{G}\left(G^{\circ}\right)$. Now $E=C_{G}\left(G^{\circ}\right)$ is the desired group.

This completes the proof of Theorem 5 .

The reader might however wonder whether one really needs the big gun of the full classification of simple groups of even type for the isomorphism (*) above. Fortunately, one can obtain this isomorphism much more directly in the case of locally ${ }^{\circ}$ solvable $^{\circ}$ groups. Here is how a baby version of the proof would work along the lines of the original papers in the simple case, even though we are not going to review all details entirely.

We thus have a simple locally ${ }^{\circ}$ solvable $^{\circ}$ group $H$ of finite Morley rank of even type, and we want to show that $H \simeq \operatorname{PSL}_{2}(K)$ for some algebraically closed field $K$ of characteristic 2 .

Fix $U$ a maximal 2-unipotent subgroup of $H$. Let $B=N^{\circ}(U)$ and $M=$ $N(U)=N(B)$. We know that $B$ is a Borel subgroup of $H$ by DJ07a, Lemma 3.9]. One sees easily that $M$ is weakly embedded in $H$, which means that it is a proper definable subgroup containing an infinite Sylow 2-subgroup and such that $M \cap M^{h}$ has finite Sylow 2-subgroups for any element $h$ of $H$ not in $M$ ABC99. Actually the strict inclusion $M<H$ follows from the nonsolvability of the ambient locally ${ }^{\circ}$ solvable $^{\circ}$ group $H$ (see [DJ07a, Lemma 3.7]). The property of finiteness of Sylow 2-subgroups of intersections of distinct conjugates of $M$ follows from specific Uniqueness Theorems, analogous to those of the so-called Bender Method in finite group theory, available in the context of locally ${ }^{\circ}$ solvable $^{\circ}$ groups of finite Morley rank, DJ07a, Corollary 4.4] or DJ07a, Corollary 5.12].

One sees easily with the same kind of arguments that for any nontrivial 2unipotent subgroup $V$ of $U, N^{\circ}(X) \leq M$ for any infinite definable subgroup $X$ of $C^{\circ}(V)$. This is because $N^{\circ}(X)$ is solvable by local ${ }^{\circ}$ solvability ${ }^{\circ}$, and thus one can use the Uniqueness Theorems of [DJ07a, §4.1], more specifically [DJ07a, Corollary 4.4] according to which $B$ is the unique Borel subgroup containing any of its nontrivial 2-unipotent subgroups.

This shunts the most difficult part of the analysis, Jal01b, Théorème 4.1], reducing essentially to the situation of [Jal01b, §3], with $M^{\circ}=B$ solvable by local $^{\circ}$ solvability $^{\circ}$ (a rather undirect fact in the general case of $\mathrm{ABC} 08$, but rather direct in the case of [Jal01b]). We leave to the reader the pleasure of accomplishing the final recognition of $\mathrm{PSL}_{2}$ along the lines of arguments and the computations of [Jal01b, Théorème 3.1], using here the fact that all normalizers ${ }^{\circ}$ of nontrivial solvable infinite subgroups are solvable. 


\subsection{Locally $^{\circ}$ solvable $^{\circ}$ groups of mixed type}

A corollary of the full classification of simple groups of finite Morley rank of even type $\mathrm{ABC08}$ and of the arguments of [Jal99] is the following.

Fact 9 [ABC08] There is no simple group of finite Morley rank of mixed type.

We obtain a similar result for connected locally ${ }^{\circ}$ solvable $^{\circ}$ groups of finite Morley rank replacing the simplicity assumption by a mere nonsolvability assumption, which is best stated in the following form.

Theorem 10 Let $G$ be a locally ${ }^{\circ}$ solvable $e^{\circ}$ group of finite Morley rank of mixed type. Then $G^{\circ}$ is solvable.

Theorem 10 can be deduced as a special case of the general theory developed in $\mathrm{ABC08}$, and at the end of the present section we will review how this can be done. In any case, the core of the proof boils down in our special context to the argument below.

To prove directly Theorem 10, we assume toward a contradiction that $G$ is a connected counterexample of minimal rank to the above statement.

As $G$ is not solvable, it has a finite solvable radical by local ${ }^{\circ}$ solvability ${ }^{\circ}$. Dividing by the latter, one gets a group still of mixed type, still locally ${ }^{\circ}$ solvable $^{\circ}$ but now semisimple ([DJ07a, Lemma 3.15]), and in which all proper nonsolvable definable connected subgroups are not of mixed type by minimality.

Fix $U$ a maximal 2-unipotent subgroup of $G$. The first main claim is

$$
M:=N(U) \text { is weakly embedded in } G .
$$

This is a special application of [DJ07a, Corollary 5.12] for the prime $p=2$. We also note that for this particular prime $p=2$ it is necessary that a nontrivial 2 -torus commutes with a nontrivial 2-unipotent subgroup by Fact 11 so that the general strategy developed in the simple case [ABC97] also works here, as explained after [DJ07a, Corollary 5.12]. It provides in particular a proof very similar to the one used in the simple case: $M=N(U)=N\left(U^{\perp}\right)$ where $U^{\perp}=T_{2}(C(U))$, and one checks easily that $M$ contains the normalizer of each of its nontrivial 2-unipotent subgroups (By the Uniqueness Theorem of [DJ07a] ) and similarly for its 2-tori $T: N^{\circ}(T)$ is solvable by local ${ }^{\circ}$ solvability ${ }^{\circ}$, contains $U$, and the Uniqueness Theorem applies again.

The next point is the following remark.

Fact 11 [Jal99, Fait 2.18] Let $G$ be a group of finite Morley rank, $S$ a Sylow 2 -subgroup of $G, T$ the maximal 2-torus of $S^{\circ}$, and $t$ an element of $T$. Then $t^{G} \cap S^{\circ}$ is contained in $T$, and is in particular finite.

Proof. The first claim follows from an argument of control of fusion in $p$-tori by their normalizers, which has been known for a long time for the particular prime $p=2$ [BN94, Lemma 10.22], and the present formulation can be tracked in [Alt96, Fact 2.48]. Anyway we refer to [DJ07a, Corollary 2.20] for the most 
general formulation of such arguments of control of fusion, in a form which directly applies here.

The finiteness of $t^{G} \cap S^{\circ}$ follows, as the 2-torus $T$ has only finitely many elements of order $2^{n}$ for each $n$ [BP90.

A proper definable subgroup $M$ is strongly embedded if it has nontrivial Sylow 2-subgroups and $M \cap M^{g}$ has trivial Sylow 2-subgroups for any element $g$ of $G$ not in $M$. There is a much similar notion in the finite case, used notably by Bender, and the notion of weak embedding is its neoclassical revival in the case of groups of finite Morley rank.

The next point is then the following.

Lemma $12 M$ is not strongly embedded in $G$.

Proof. Assume towards a contradiction $M$ strongly embedded. Then its involutions would necessarily be conjugate [BN94, Theorem 10.19]. In particular an involution of a maximal 2-torus $T$ of $M$ would be conjugate to any involution of $U$. But there are infinitely many involutions in 2-unipotent groups BP90, and this contradicts Fact 11.

By general results on strong/weak embedding (see [ABC08, Part B]), one then concludes that there exists an involution $\alpha$ of $M$ which is problematic in the sense that

$$
C(\alpha) \not \leq M
$$

and the next step consists in applying Theorem 5 to its centralizer ${ }^{\circ}$.

Lemma 13 Let $\alpha$ be a problematic involution of $M$. Then $C^{\circ}(\alpha) \simeq \operatorname{PSL}_{2}(K)$ for some algebraically closed field $K$ of characteristic 2 .

Proof. The involution $\alpha$ normalizes $U=U_{2}(B)$, as connected solvable groups of finite Morley rank have only one maximal $p$-unipotent subgroup (see for example [DJ07a, Fact 2.15] for the most general and contemporary version of this, or Nes90b for the oldest results from which it can be deduced). As infinite nilpotent-by-finite $p$-groups of finite Morley rank contain infinitely many central elements of order $p$ BP90, $\alpha$ centralizes a nontrivial 2-unipotent subgroup of $U$.

We claim that $C^{\circ}(\alpha)$ is not solvable. This follows from the Uniqueness Theorems of [DJ07a, §4.1], according to which a nontrivial $p$-unipotent subgroup of any locally ${ }^{\circ}$ solvable $^{\circ}$ group of finite Morley rank is contained in a unique Borel subgroup. In particular $B$ is the unique Borel subgroup containing $C_{U}^{\circ}(\alpha)$. Assuming $C^{\circ}(\alpha)$ solvable, we would get in particular $C^{\circ}(\alpha) \leq B$, and $C(\alpha) \leq$ $N\left(C_{U}^{\circ}(\alpha)\right) \leq N(B)=M$, as $B$ is the unique Borel subgroup containing $C_{U}^{\circ}(\alpha)$, a contradiction since $\alpha$ is problematic.

By semisimplicity of $G, C^{\circ}(\alpha)<G$, and by minimality, $C^{\circ}(\alpha)$ cannot be of mixed type. As it contains a nontrivial 2-unipotent subgroup as just seen, 
$C^{\circ}(\alpha)$ is a locally ${ }^{\circ}$ solvable ${ }^{\circ}$ group of even type. Now Theorem 5 yields the desired isomorphism type of $C^{\circ}(\alpha)$.

For the final step we are now in a position to conclude as at the end of Jal99, using the same relevant technical lemma on involutions isolated to tackle the configuration appearing.

Fact 14 [Jal99, Lemme 4.1] Let $G$ be a group of finite Morley rank, with involutions $i, t, \alpha$, and $\alpha^{\prime}$ satisfying the following five conditions.

(1) $i$ and $t$ are not conjugate.

(2) $U_{2}(C(\alpha)) \simeq \mathrm{PSL}_{2}(K)$ for some algebraically closed field $K$ of characteristic 2 .

(3) $\alpha^{\prime}$ is the unique involution of the definable hull $H(i t)$ of it ( $i \cdot t$, not it).

(4) $i \in U_{2}(C(\alpha)), t \in C(\alpha)$.

(5) $\alpha^{\prime} \notin U_{2}(C(\alpha))$.

Then $t \alpha^{\prime} \in U_{2}(C(\alpha))$.

Proof of Theorem 10, We keep the previous notations.

Applying DJ07a Lemma 3.35 (2)] to the conjugacy class of a 2-toral involution $t$ of $G$, one gets as $G$ is not solvable that $t^{G} \cap M$ is not generic in $t^{G}$ (notice that $t^{G}$ is infinite as $C(t)<G$ and $G$ is connected). In particular one may assume, choosing a suitable conjugate of $t$, that $t$ is a 2 -toral involution not in $M$.

Let $\alpha_{0}$ be a problematic involution of $M$. As seen in the proof of Lemma 13. there exists an involution $i_{0}$ in $C_{U}^{\circ}\left(\alpha_{0}\right)$. As involutions of the latter group are all conjugate in $C^{\circ}\left(\alpha_{0}\right)$ by Lemma 13, Corollary 11]implies that $t$ and $i_{0}$ are not conjugate. In particular there exists an involution $\alpha$ in the definable hull $H\left(i_{0} t\right)$ of $i_{0} t$ [BN94, Proposition 10.2]. We have

$$
\left[i_{0}, \alpha\right]=1 \text { and }[t, \alpha]=1
$$

Lemma 13 implies in particular that problematic involutions of $M$ never belong to a unipotent subgroup, as they cannot centralize a nontrivial 2-torus. In particular $i_{0}$ is not problematic in $M$, i.e., $C\left(i_{0}\right) \leq M$. The first commutation implies thus that $\alpha$ is in $M$, and as $t \notin M$ the second commutation implies that $\alpha$ is a problematic involution of $M$.

Take an involution $i$ in $C_{U}^{\circ}(\alpha)$. Again $i$ and $t$ are not conjugate, hence there is an involution $\alpha^{\prime}$ in $H(i t)$. Similarly, $\alpha^{\prime}$ commutes with $i$ and $t$, and is thus a problematic involution of $M$.

One can check that the five conditions of Fact 14 are met for these involutions $i, t, \alpha$, and $\alpha^{\prime}$. For the third for example, we note that $H(i t)$ contains no 
nontrivial 2-torus, as $\alpha^{\prime} \in H(i t)$ and by the structure of centralizers of problematic involutions. For the last point, this is by the fact that problematic involutions cannot belong to a 2-unipotent subgroup. The conclusion of Fact 14 is then

$$
t \alpha^{\prime} \in U_{2}(C(\alpha)) .
$$

As $t$ centralizes $\alpha$, it acts on $C^{\circ}(\alpha)$, and by the structure of the latter the action must be by inner automorphism. Since $t$ centralizes $t \alpha^{\prime}$, it centralizes the Sylow 2-subgroup $A_{t \alpha^{\prime}}$ of $C^{\circ}(\alpha)$ containing $t \alpha^{\prime}$.

Now $A_{t \alpha^{\prime}} \leq C(t)<G$, and hence by our minimality assumption $C(t)$ is of even type. But $t$ belongs to a nontrivial 2-torus, and in particular centralizes it. This is a contradiction which ends the proof of Theorem 10.

Another approach to Theorem 10 would be to use the fact that

$$
\left[T_{2}(G), U_{2}(G)\right]=1 .
$$

This is a general corollary of the full classification of simple groups of finite Morley rank of even type ABC08, Lemma V 2.3, Theorem X 1, Chap. V Mixed Type $L^{*}$ Theorem]. With this commutation, one concludes easily to the solvability of $G$ when both factors in the commutator are nontrivial, as both the normal factors are then solvable by local ${ }^{\circ}$ solvability ${ }^{\circ}$ of $G$. If one tries to prove directly this commutation in our case, then as in [ABC08, Lemma V 2.3] one may want to look at the action of 2-tori on $U_{2}(G)$. As in Section 1.1 or ABC08, Proposition II 6.2, Lemma II 6.3], one is then interested into the socle of $U_{2}(G)$ modulo $R\left(U_{2}(G)\right)$, a direct product of connected simple factors. If no such factor is of mixed type, then one can conclude that $\left[T_{2}(G), U_{2}(G)\right]=1$ as in [ABC08, Lemma V 2.3]. Otherwise, the situation reduces to the case where $G$ is a simple group such that $G=U_{2}(G)=T_{2}(G)$, and in our case this was disposed of by the core of the proof given above.

As it is was explained in [DJ07a, §3.3], there might be serious obstructions if one wants a version of Theorem 10 for primes different from 2.

We now note that Theorem 4 follows from Theorems 5 and 10. By-the-by, we mention the following corollary of Theorem 4 on connectedness of Sylow 2-subgroups in small groups of finite Morley rank.

Corollary 15 Let $G$ be a connected locally ${ }^{\circ}$ solvable ${ }^{\circ}$ group of finite Morley rank with a nonconnected Sylow 2-subgroup. Then $G$ is nonsolvable and of odd type.

Proof. It is known that Hall $\pi$-subgroups are connected in any connected solvable group of finite Morley rank and for any set $\pi$ of primes. In PSL ${ }_{2}$ over some algebraically closed field $K$ of characteristic 2, Sylow 2-subgroups are also connected. It can be seen either by the transitivity of the action on them of their normalizers or by the fact that they are definably isomorphic to the additive group of the ground field $K$, which is interpretable and of finite Morley rank.

It follows that the only remaining possibility in Theorem 4 is case (3) in that theorem. 


\subsection{Involutive actions}

The structure of nonsolvable locally ${ }^{\circ}$ solvable $^{\circ}$ groups of odd type, left undetermined in Theorem 4 will be considered in DJ07b as mentioned in the introduction. Now we merely deal with specialized topics concerning groups of odd type in general and which will in particular be applied in the lengthy analysis of [DJ07b].

Before moving ahead, we concentrate in general on definable involutive automorphisms of groups of finite Morley rank. When $\alpha$ is an automorphism of a group $G$, we let

$$
G^{+\alpha}=\left\{g \in G \mid g^{\alpha}=g\right\} \text { and } G^{-\alpha}=\left\{g \in G \mid g^{\alpha}=g^{-1}\right\} .
$$

Both sets are definable whenever $\alpha$ is a definable automorphism of $G$. In general, only the centralizer $G^{+\alpha}$ of $\alpha$ in $G$ is necessarily a subgroup of $G$. When there is no risk of confusion between different possible automorphisms $\alpha$, we will sometimes omit the subscript $\cdot_{\alpha}$ in the notation of the two sets as above, and thus just speak of $G^{+}$and $G^{-}$.

We start by mentioning the older results on involutive definable automorphisms $\alpha$ of groups of finite Morley rank in terms of $G^{+}$and $G^{-}$.

Fact 16 [Nes90a] Let $\alpha$ be a definable involutive automorphism of a group $G$ of finite Morley rank. If $\alpha$ fixes only finitely many elements in $G$, then $G$ has a definable (abelian) subgroup of finite index inverted by $\alpha$, i.e., $G^{\circ} \subseteq G^{-}$.

Fact 17 [BN94, Ex. 14, p. 73] Let $G$ be a group of finite Morley rank without involutions and $\alpha$ a definable involutive automorphism of $G$. Then $G^{-}$ is 2 -divisible and one has a decomposition $G=G^{+} \cdot G^{-}$, where the corresponding multiplication map is one-to-one.

In the present section we are essentially going to give a generalization of Fact 17 when the group $G$ contains a central and divisible Sylow 2-subgroup. We first note the following general lemma.

Lemma 18 Let $G$ be a group of finite Morley rank whose Sylow p-subgroup is a central p-torus. Then $G$ is p-divisible.

Proof. Let $g$ be an arbitrary element of $G$. Then the definable hull $H(g)$ of the cyclic group $\langle g\rangle$ can be written as

$$
H(g)=\Delta \oplus\langle\zeta\rangle
$$

where $\Delta$ is $p$-divisible and $\zeta$ a $p$-element, by using [ABC08, Lemma 2.16] and by decomposing the finite cyclic part into its $p$-primary component and a (cyclic) complement. Let $h$ be a $p$-th root of $g \zeta^{-1}$ in $\Delta$. Let $\eta$ be a $p$-th root of $\zeta$ in the Sylow $p$-subgroup. As $\eta$ is central in $G$, one gets

$$
g=g \zeta^{-1} \zeta=h^{p} \eta^{p}=(h \eta)^{p} .
$$


In general, a group of even type is not 2-divisible. One may wonder whether groups of odd type are 2-divisible, but this need not hold in general neither as the following example shows. If $B$ is a Borel subgroup of $\mathrm{SL}_{2}(K)$, with $K$ an algebraically closed field of characteristic different from $2, u$ a nontrivial unipotent element in $B$, and $i$ the unique (central) involution of $\mathrm{SL}_{2}(K)$, then $u i$ is not a square in $\mathrm{SL}_{2}(K)$. In particular $B$ is a connected 2-step solvable group of finite Morley rank of odd type, and $B$ is not 2-divisible!

Our generalization of Fact 17 is the following.

Theorem 19 Let $G$ be a group of finite Morley rank whose Sylow 2-subgroup is a (possibly trivial) central 2-torus, and $\alpha$ a definable involutive automorphism of $G$. Then $G=G^{+} \cdot G^{-}$where the fibers of the associated product map are finite. Furthermore one also has $G=\left(G^{+}\right)^{\circ} \cdot G^{-}$whenever $G$ is connected.

The proof of Theorem 19 generalizes that of Fact 17. We note that Theorem 19 also incorporates [Del08b, Fait 1.19]. We shall need the following intermediate general lemma whose proof has a flavor similar to that of Jal01b, Lemme 4.45].

Lemma 20 Let $G$ be a group of finite Morley rank whose Sylow 2-subgroup is central. If $a^{b}=a^{-1}$ for two elements $a$ and $b$ of $G$, then $a$ is either the identity or an involution of $G$.

Proof. The set $C^{ \pm}(a)=\left\{x \in G, a^{x}=a^{ \pm 1}\right\}$ is a definable subgroup of $G$. As $C^{ \pm}(a) / C(a)$ has exponent at most 2, there is a (possibly trivial) 2-element in any coset of $C(a)$ in $C^{ \pm}(a)$ by [ABC08, Lemma 2.18]. As 2-elements are central by assumption, this proves that any coset of $C(a)$ in $C^{ \pm}(a)$ is in $C(a)$, and thus $C^{ \pm}(a)=C(a)$. In particular $a=a^{-1}$, and $a^{2}=1$.

Proof of Theorem 19. Recall that $G$ is a group of finite Morley rank whose Sylow 2-subgroup is a (possibly trivial) central 2-torus, and that $\alpha$ is an automorphism of $G$ of order at most 2 .

Step 1. If $[g, \alpha]$ is a non-trivial 2-element, then there exists $h$ in $G$ such that $[g, \alpha]=[h, \alpha]^{2}$.

Proof. Assume $\zeta=[g, \alpha]$ has order $n=2^{k}$ for some $k$. As $G$ is 2-divisible by Lemma 18, there exists an element $h$ in $G$ such that $h^{2}=g$. Now $\zeta=[g, \alpha]=$ $\left[h^{2}, \alpha\right]=[h, \alpha]^{h}[h, \alpha]$ is central and has order $n$, so $h$ inverts $q=[h, \alpha]^{n}$. By Lemma 20, $q$ has order at most 2. In particular $[h, \alpha]$ is a 2-element, and it is in particular central in $G$. One then gets $[g, \alpha]=[h, \alpha]^{h}[h, \alpha]=[h, \alpha]^{2}$.

The moral of Step 1 is that in general $G^{-}$needs not be 2-divisible as in Fact 17. but its subset $\{[g, \alpha], g \in G\}$ is 2-divisible as we will see in the course of the next step. 
Step 2. $G=G^{+} \cdot G^{-}$.

Proof. Let $g \in G$. One can write the definable hull $H([g, \alpha])$ of $[g, \alpha]$ as in Lemma 18 as $H([g, \alpha])=\Delta \oplus\langle\zeta\rangle$, where $\Delta$ is a 2-divisible group and $\zeta$ is a 2element. Let $h$ be a square root of $[g, \alpha] \zeta^{-1}$ in $\Delta$. Notice that $h$ centralizes $[g, \alpha]$ and is inverted by $\alpha$. Hence $[g h, \alpha]=[g, \alpha]^{h}[h, \alpha]=[g, \alpha] h^{-2}=\zeta$. By Step 1, there is $x \in G$ such that $\zeta=[x, \alpha]^{2}$. So $\eta=[x, \alpha]$ is a 2-element inverted by $\alpha$, whose square is $\zeta$. As $\eta \in Z(G)$, we find that $\eta^{-1} h^{-1}$ is inverted by $\alpha$. Moreover, $[g h \eta, \alpha]=[g, \alpha]^{h \eta}[h, \alpha]^{\eta}[\eta, \alpha]=h^{2} \zeta h^{-2} \eta^{-2}=1$, so $g=(g h \eta)\left(\eta^{-1} h^{-1}\right)$ is a suitable decomposition.

Step 3. If $a x=b y$ with $a, b \in G^{+}$and $x, y \in G^{-}$, then $a^{-1} b$ is the identity or an involution fixed by $\alpha$. Hence the fibers of the product decomposition as in Step 2 are of cardinal at most $\left|\left\{k \in G \mid k^{2}=1\right\}\right|$.

Proof. We have

$$
\left(a^{-1} b\right)^{y}=\left(x y^{-1}\right)^{y}=y^{-1} x=\alpha\left(y x^{-1}\right)=\alpha\left(b^{-1} a\right)=b^{-1} a=\left(a^{-1} b\right)^{-1},
$$

so $y$ inverts $a^{-1} b$. By Lemma 20, $a^{-1} b$ has order at most 2. In particular, $a^{-1} b$ lies in the central elementary abelian 2-subgroup of $G$, and it is fixed by $\alpha$. We also note that the central elementary abelian 2-subgroup of $G$ is exactly $\left\{k \in G \mid k^{2}=1\right\}$.

Step 4. Left $G^{+}$-translates of the set $\left(G^{+}\right)^{\circ} \cdot G^{-}$are disjoint or equal.

Proof. Assume that $a\left(G^{+}\right)^{\circ} \cdot G^{-}$meets $b\left(G^{+}\right)^{\circ} \cdot G^{-}$, in say $a g_{+} g_{-}=b h_{+} h_{-}$ with natural notations. By Step $3, z=\left(a g_{+}\right)^{-1}\left(b h_{+}\right)$is central and fixed by $\alpha$. So $z=\left(b h_{+}\right)\left(a g_{+}\right)^{-1}$. Hence for any $b \gamma_{+} \gamma_{-} \in b\left(G^{+}\right)^{\circ} \cdot G^{-}$one finds $b \gamma_{+} \gamma_{-}=z z^{-1} b \gamma_{+} \gamma_{-}=a\left(g_{+} h_{+}^{-1} \gamma_{+}\right)\left(\gamma_{-} z\right)$, which lies in $a\left(G^{+}\right)^{\circ} \cdot G^{-}$. The converse inclusion holds too.

Step 5. Exactly $\operatorname{deg}(G)$ left $G^{+}$-translates of $\left(G^{+}\right)^{\circ} \cdot G^{-}$cover $G$. In particular, if $G$ is connected, then $G=\left(G^{+}\right)^{\circ} G^{-}$.

Proof. We consider such left translates. They all have $\operatorname{rank} \operatorname{rk}(G)$ by Step 3 . As they are disjoint or equal by Step 4 , exactly $\operatorname{deg}(G)$ of them suffice to cover $G$.

This ends the proof of Theorem 19 .

The following results are not used here, but it is worth mentioning them for the sake of completeness of the present section. The first one is an important commutation principle, and the second one is a downward invariance result.

Fact 21 [Del07a, Lemme 3.1] Let $G$ be a group, $H$ and $K \leq N(H)$ two subgroups with $K$ 2-divisible. Suppose that there is an involution $i$ in $G$ which inverts $K$ and centralizes or inverts $H$. Then $[H, K]=1$. 
Fact 22 (Compare with [Del07a, Fait 3.12]) Let $G$ be a group of finite Morley rank, $K \leq G$ a definable 2-subgroup normalizing a definable subgroup $H$ of $G$, and $S$ a Sylow 2-subgroup of $H$. Then an $H$-conjugate of $K$ normalizes $S$.

Proof. Consider the definable group $H K$, and let $\hat{S}$ denote a Sylow 2-subgroup of $H K$ containing $S$. By conjugacy of Sylow 2-subgroups, $K^{\gamma} \leq \hat{S}$ for some $\gamma$ in $H K$, and in fact $\gamma$ may be chosen in $H$. Now the $H$-conjugate $K^{\gamma}$ of $K$ normalizes $\hat{S} \cap H=S$.

\subsection{Solvable groups of odd type}

Since the original unpublished Jal00 on small groups of odd type, most of the work has consisted in developing certain arguments based on considerations involving strongly real elements, that is products of two involutions. The goal is ultimately to adapt to odd type groups such arguments from finite group theory, first imported by Nesin to groups of finite Morley rank.

In $\mathrm{PSL}_{2}(K)$, with $K$ an algebraically closed field of characteristic different from 2, the standard Borel subgroup contains two kinds of strongly real elements. Those of maximal unipotent subgroups on the one hand are inverted by involutions inside their respective Borel subgroups, and those in maximal tori on the other hand are inverted by involutions outside the Borel subgroup, and corresponding to liftings of elements of the Weyl group. We may call these strongly real elements "insiders" and "outsiders" respectively.

When working with small groups of odd type, most complications arise when trying to get a control on outsiders, that is strongly real elements inside a Borel subgroup but such that the two involutions forming the product are outside this Borel subgroup. This is typically done for a standard Borel subgroup, that is a Borel subgroup $B$ containing the centralizer ${ }^{\circ}$ of a particular involution. In this case outsiders of this Borel subgroup $B$, inverted by a fixed involution, are expected to form a torus of $B$ in the expected group $\mathrm{PSL}_{2}$. Other complications arise when one has to compare insiders and outsiders of such a Borel subgroup, and in this case one also occasionally needs in this delicate work a good understanding of insiders. We refer to [DJ07b] anyway.

The study of insiders merely boils down to the study of connected solvable groups of odd type, and this is the purpose of the present section. Recall first from BP90 that the connected component of the Sylow 2-subgroup of a group of finite Morley rank is a direct product of finitely many copies of the quasicyclic group $\mathbb{Z}_{2^{\infty}}$, with this finite number called the Prüfer 2-rank of the group. As connected solvable groups of finite Morley rank have connected Sylow 2 -subgroups, the Sylow 2-subgroup $S$ of a connected solvable group of odd type satisfies

$$
S=S^{\circ} \simeq \mathbb{Z}_{2^{\infty}} \times \cdots \times \mathbb{Z}_{2^{\infty}}
$$

where the number of factors involved is the Prüfer 2-rank. 
Before moving ahead, we recall some more general background. As for the solvable radical, there is a similar notion of Fitting subgroup in any group $G$ of finite Morley rank [BN94, Theorem 7.3], where nilpotence replaces solvability, usually denoted by $F(G)$. A Carter subgroup of a group of finite Morley rank is a definable connected nilpotent subgroup of finite index in its normalizer, and it is nontrivial but a fact that any group of finite Morley rank contains a Carter subgroup [FJ05].

Lemma 23 Let $G$ be a connected group of finite Morley rank without nontrivial $p$-unipotent subgroups for some prime $p$, and $t$ a p-element of $G$. Then

(1) The element $t$ belongs to a Carter subgroup $Q$ of $G$, and $Q \leq C^{\circ}(t)$.

(2) If furthermore $G=N Q$ for some normal definable subgroup $N$, then $G=$ $C^{\circ}(t) N$.

Proof. (1). By the main result of [BC07, $t$ belongs to a $p$-torus, say $T_{p}$. Now any decent torus is contained in a Carter subgroup by the construction given in [FJ05. We have thus $t \in T_{p} \leq Q$ for some Carter subgroup $Q$ of $G$, and as any connected nilpotent group of finite Morley rank has a unique decent torus which is central, by earlier work of Nesin, one concludes that $Q \leq C^{\circ}\left(T_{p}\right) \leq C^{\circ}(t)$. The reader can also consult [Jal08a, Fact 4] for similar facts in this direction.

(2). We have $G=Q N \leq C^{\circ}(t) N \leq G$, and thus $G=C^{\circ}(t) N$.

Here are the main lemmas used in DJ07b concerning strongly real elements inside connected solvable groups of odd type. We stress the fact that the Prüfer 2-rank is not 1 in general, a technical complication which has to be entirely taken into account in Del08b and in DJ07b. For an element normalizing a subgroup we confound below the element with the automorphism it induces by conjugation in the notation introduced at the begining of Section 1.3 .

Lemma 24 (Inner Computation) Let $B$ be a connected solvable group of finite Morley rank of odd type and let $j$ be an involution of $B$. Then

(1) $B=C_{B}^{\circ}(j) \cdot\left[F^{\circ}(B)\right]^{-j}$, where the fibers of the associated product map are finite. In particular $\mathrm{rk}(B)=\operatorname{rk}\left(C_{B}^{\circ}(j)\right)+\operatorname{rk}\left(\left[F^{\circ}(B)\right]^{-j}\right)$.

(2) For any definable subgroup $U$ of $Z\left(F^{\circ}(B)\right)$ normal in $B$, the subgroup $U^{-j}$ is normal in $B$.

Proof. (1). As $B$ is of odd type, there are finitely many involutions in the connected nilpotent group $F^{\circ}(B)$ BN94, §6.2].

We claim that the fibers of the multiplication map

$$
P: C^{\circ}(j) \times\left(F^{\circ}(B)\right)^{-j} \rightarrow B
$$

are finite. If $c f=c^{\prime} f^{\prime}$, with obvious notations, then $c^{-1} c=f^{\prime} f^{-1}$, and this element is centralized by $j$. Hence one gets $f^{\prime} f^{-1}=\left(f^{\prime} f^{-1}\right)^{j}=f^{\prime-1} f$, and 
$f^{\prime 2}=f^{2}$. The nilpotent group $F^{\circ}(B)$ has the form $T * U$, a central product with finite intersection, where $T$ denotes the maximal decent torus of $F^{\circ}(B)$ and $U$ is a definable connected nilpotent subgroup. In particular all the 2-torsion of $F^{\circ}(B)$ is in $T$. In $F^{\circ}(B)$ modulo $T$ one also has $f^{\prime 2}=f^{2}$ in this quotient. Now in groups of finite Morley rank without involutions any element has a unique square root ([BN94, p. 72], [ABC97, Fact 2.25]), and one concludes that $f=f^{\prime}$ modulo $T$, i.e., $f^{\prime}=f t$ for some $t$ in $T$. As $T$ is central in $F^{\circ}(B)$, one gets by taking squares that $t^{2}=1$, and as $T$ has only finitely many involutions it implies that there are only finitely many possibilities for $f^{\prime}$, once $f$ is fixed. This gives the desired finiteness of the fibers of the multiplication map $P$.

So our statement reduces to proving that the map $P$ is onto.

In connected solvable groups $B$ of finite Morley rank, Carter subgroups are conjugate and cover the connected nilpotent quotient $B / F^{\circ}(B)$ by [Nes90b] and [FJ08, Theorem 3.11 and Proposition 5.1]. Combined with Lemma23 this yields

$$
B=C^{\circ}(j) F^{\circ}(B)
$$

and for our desired factorisation it suffices thus to show that

$$
F^{\circ}(B)=C_{F^{\circ}(B)}^{\circ}(j) \cdot\left[F^{\circ}(B)\right]^{-j} .
$$

But the Sylow 2-subgroup of $F^{\circ}(B)$ is a central 2-torus, again by earlier results of Nesin according to which divisible torsion is central in connected nilpotent groups of finite Morley rank. Hence Theorem 19 can be applied with the action by conjugation of $j$ on $F^{\circ}(B)$, and this gives exactly the desired factorisation $(\dagger)$ as above.

(2). If $c \in C^{\circ}(j)$ and $u \in U^{-j}$, then $\left(u^{c}\right)^{j}=u^{c j}=u^{j c}=u^{-c}=\left(u^{c}\right)^{-1}$, and as $u^{c} \in U$ by normality of $U$ in $B$ one gets $u^{c} \in U^{-j}$. Hence the subgroup $U^{-j}$ is normalized by $C^{\circ}(j)$, and as it is also central in $F^{\circ}(B)$ it is normal in $B=C^{\circ}(j) F^{\circ}(B)$.

The following application of Lemma 24 will be used in the most critical situations in DJ07b. In the second claim of the corollary below the two involutions considered are not necessarily conjugate, a point we will use fully in the analysis of DJ07b].

Corollary 25 Let $G$ be a group of finite Morley rank, $i$ an involution of $G$, and assume $C^{\circ}(i) \leq B$ for some definable connected solvable subgroup $B$ of odd type. Let $j$ be an involution of $B$. Then

(1) $\operatorname{rk}(B)=\operatorname{rk}\left(C_{B}^{\circ}(j)\right)+\operatorname{rk}\left(\left[F^{\circ}(B)\right]^{-j}\right)$.

(2) In particular, if $C^{\circ}(j) \leq B$ and $C^{\circ}(i)$ and $C^{\circ}(j)$ have the same rank (which is the case in particular if $i$ and $j$ are conjugate), then $\operatorname{rk}\left(\left[F^{\circ}(B)\right]^{-j}\right)=$ $\operatorname{rk}\left(\left[F^{\circ}(B)\right]^{-i}\right)$. 
Proof. By assumption, $C^{\circ}(i)$ contains trivial 2-unipotent subgroups. It follows from the torality result of $\mathrm{BC} 07$ ] that $i$ belongs to a 2 -torus, say $T$. Then $i \in T \leq C^{\circ}(i) \leq B$. Now Lemma 24 applies in $B$.

We also mention the following additional information to Lemma 24 when the Prüfer 2-rank is 1. Of course, this corresponds more and more to abstract descriptions of the Borel subgroups of $\mathrm{PSL}_{2}$ or $\mathrm{SL}_{2}$ over algebraically closed fields of characteristic different from 2.

Lemma 26 Adopt the same assumptions and notations as in Lemma 24 and assume furthermore that the Prüfer 2-rank is 1 . Then

(1) $C^{\circ}(j)<B$ if and only if $F(B)$ contains no involutions.

(2) $C_{B}(j)$ is connected, $B=C^{\circ}(j) \cdot\left[F^{\circ}(B)\right]^{-j}$, and the multiplication map giving this decomposition is one-to-one whenever $C^{\circ}(j)<B$.

(3) The set of involutions of $B$ is exactly $j^{F^{\circ}(B)}$.

Proof. First, we note that a connected solvable group of finite Morley rank has connected Sylow 2-subgroups. In particular a connected solvable group of finite Morley rank of odd type and of Prüfer 2-rank 1 has Sylow 2-subgroups isomorphic to $\mathbb{Z}_{2 \infty}$.

(1). If $F^{\circ}(B)$ contains an involution, its maximal 2-torus is nontrivial. As it is central in $B$ by [DJ07b, Fact $2.12(1)$ ], the toral involution is central in $B$, and it must be $j$. If the Sylow 2-subgroup of $F(B)$ is finite, then it is central in $B$ by [DJ07b, Fact 1.2]. One gets again that an involution of $B$ must be central in $B$ and by connectedness of the Sylow 2-subgroups of $B$ it must be $j$ again.

Conversely, if $C_{B}(j)=B$, then $F(B)$ contains the involution $j$.

(2). We can prove the factorisation much more directly here. By (1), we may assume $F^{\circ}(B)$ without involutions. We work in $B / F^{\circ}(B)$. As this quotient is abelian by [Nes90b], $j$ induces by conjugation a trivial action on this quotient.

In particular, for every $b \in B$, there exists $f \in F^{\circ}(B)$ such that $b^{j}=b f$. Conjugating again by $j$ one gets $f^{j}=f^{-1}$, so $f \in\left[F^{\circ}(B)\right]^{-j}$. By (1) and Fact 17. $f=g^{2}$ for some $g$ still in $\left[F^{\circ}(B)\right]^{-j}$. Then $b=(b g) g^{-1}$, where $(b g)^{j}=$ (bf) $g^{-1}=b g \in C_{B}(j)$ and $g \in\left[F^{\circ}(B)\right]^{-j}$.

Hence $B=C_{B}(j) \cdot\left[F^{\circ}(B)\right]^{-j}$. We show the uniqueness of this decomposition whener $C^{\circ}(j)<B$. If $c_{1} f_{1}=c_{2} f_{2}$, with natural notations, then $f_{2} f_{1}^{-1} \in C(j)$, and $f_{1}^{2}=f_{2}^{2}$, which in the group without involutions $F^{\circ}(B)$ implies $f_{1}=f_{2}$, and then the uniqueness of the decomposition follows.

The product map corresponding to the decomposition $B=C_{B}(j) \cdot\left[F^{\circ}(B)\right]^{-j}$ has finite fibers. Hence each definable generic subset of the source set has a generic image in $B$. When the product map is one-to-one, this forces that $C_{B}(j)$ has Morley degree one. Otherwise, $C_{B}(j)=B$ is also connected.

(3). As the Prüfer 2-rank is 1, all involutions of the connected solvable group $B$ are conjugate by the structure of Sylow 2 -subgroups. Then (2) gives the desired equality. 


\subsection{Groups of Prüfer 2-rank 1}

It is proved in [CJ04, Lemma 2.34] that if $S$ is a Sylow 2-subgroup of a group of finite Morley rank of odd type and of Prüfer 2-rank 1, and if $w$ is an involution conjugate to the unique involution $i$ of $S^{\circ}$, then $w$ cannot centralize $S^{\circ}$ unless it is $i$. From this one can deduce that such involutions $w$ which normalize $S^{\circ}$ but are not inside must invert $S^{\circ}$, and one gets in this situation a subgroup of the Sylow 2-subgroup isomorphic to that of Chevalley groups of type PSL ${ }_{2}$ over algebraically closed fields of characteristic different from 2 .

We are going to delineate entirely the structure of Sylow 2-subgroups of connected groups of odd type and Prüfer 2-rank 1, showing in any case analogy with the structure of Sylow 2-subgroups in groups of the form $\mathrm{PSL}_{2}$ or $\mathrm{SL}_{2}$ over some algebraically closed field of characteristic different from 2 or of connected solvable groups. First, we recall from the main result of [BC07] that any 2element of a connected group of finite Morley rank of odd type is 2-toral, i.e., belongs to a 2-torus. When the Prüfer 2-rank is 1 it implies by Fact 1 that all involutions are conjugate.

Proposition 27 Let $G$ be a connected group of finite Morley rank of odd type and of Prüfer 2-rank 1. Then there are exactly three possibilities for the isomorphism type of a Sylow 2-subgroup $S$ of $G$.

(1) $S=S^{\circ} \rtimes\langle w\rangle$ for some involution $w$ which acts on $S^{\circ}$ by inversion.

(2) $S=S^{\circ} \cdot\langle w\rangle$ for some element $w$ of order 4 which acts on $S^{\circ}$ by inversion.

(3) $S=S^{\circ}$.

Our proof of Proposition 27 uses the following results.

Fact 28 [BC08] Let $G$ be a connected group of finite Morley rank of odd type, and fix some Sylow 2-subgroup $S$ of $G$. Then $C_{S}\left(S^{\circ}\right)=S^{\circ}$.

Fact 29 Aut $\left(\mathbb{Z}_{2 \infty}\right) \simeq \mathbb{Z}_{2}^{\times}$. In particular the only nontrivial automorphism of finite order of $\mathbb{Z}_{2 \infty}$ is the inversion.

Proof. It is clear that Aut $\left(\mathbb{Z}_{2 \infty}\right)$ is isomorphic to the group of invertible elements of the ring $\mathbb{Z}_{2}$. But $\mathbb{Z}_{2}{ }^{\times} \simeq \mathbb{Z} / 2 \mathbb{Z} \times \mathbb{Z}_{2}$ and the right factor is torsion-free as the characteristic is 0 . In particular $\operatorname{Aut}\left(\mathbb{Z}_{2^{\infty}}\right)$ has only one nontrivial automorphism of finite order, inversion.

Lemma 30 Let $G$ be a connected group of finite Morley rank of odd type and of Prüfer 2-rank 1, and $S$ a Sylow 2-subgroup of $G$. Then $\left[S: S^{\circ}\right] \leq 2$ and elements of $S \backslash S^{\circ}$ act on $S^{\circ}$ by inversion.

Proof. By Fact 28, every element of $S \backslash S^{\circ}$ has a nontrivial action on $S^{\circ}$. (This is the original argument in [CJ04, Lemma 2.34] in the light of the torality of [BC07.) 
Now Fact 29 implies that this action is by inversion, and in particular all elements of $S \backslash S^{\circ}$ have the same action on $S^{\circ}$. By Fact 28 again one gets that there is at most one coset of $S^{\circ}$ distinct from $S^{\circ}$ in $S$, which proves our claim.

Proof of Proposition 27. Assume that $S$ is not connected. We prove that $S$ is either isomorphic to the Sylow 2-subgroup of $\mathrm{PSL}_{2}$ or to that of $\mathrm{SL}_{2}$ in characteristic different from 2.

Let $w \in S \backslash S^{\circ}$. By Lemma 30, $S=S^{\circ} \cdot\langle w\rangle, w$ inverts $S^{\circ}$, and $w^{2} \in S^{\circ}$. If $w^{2}=1$, then $S$ is obviously as in $\mathrm{PSL}_{2}$. Now suppose $w^{2} \neq 1$. As $w$ inverts $S^{\circ}$ and $w^{2} \in S^{\circ}$, one has $w^{2}=\left(w^{2}\right)^{w}=\left(w^{2}\right)^{-1}$. So $w^{2}$ is the involution of $S^{\circ}$, as in $\mathrm{SL}_{2}$.

Remarkably, the conclusion of Proposition 27 will be obtained in a very general setting in the case of connected locally ${ }^{\circ}$ solvable $^{\circ}$ groups of odd type, in the early analysis of [DJ07b. This has the effect of simplifying slightly certain arguments in the entire classification of small groups of odd type, not spectacularly as the main difficulties with strongly real elements remain throughout, but at least morally and technically, removing certain residual involutions sometimes occuring in Weyl groups. As an example of such minor but seemingly simplifications, it entirely eliminates the need for the lengthy Sections 6.1 and 6.2 in the analysis of Weyl groups in CJ04.

\subsection{The Borovik-Cartan decomposition}

In quadratic algebra, the Cartan polar form yields a decomposition $f=u$. $s$ of any automorphism $f$ of $\mathbb{C}^{n}$, with $u$ in the unitary group and $s$ a selfadjoint automorphism. It is well known by french taupins that the Cartan decomposition can be used to prove connectedness of the unitary group. Lines of arguments, formally similar, were implicitely (and unconsciously) rediscovered to prove connectedness of centralizers of involutions in certain context of groups of finite Morley rank in $\mathrm{BBC07}$. We are going to rework this in a form more suitable for DJ07b], but let us first serve some refreshments.

We denote (Hermitian) adjunction on $\mathbb{C}^{n}$ by ${ }^{*}$, so that

$$
(x y)^{*}=y^{*} x^{*} \text { and } x^{* *}=x
$$

for any automorphisms $x$ and $y$ of $\mathbb{C}^{n}$. We note that it follows from the first equality that $1^{*}=1$ and $\left(x^{-1}\right)^{*}=\left(x^{*}\right)^{-1}$ for any automorphism $x$.

If $g$ is an automorphism of $\mathbb{C}^{n}$, then $g^{*} g$ is a self-adjoint automorphism, and one can sometimes get a self-adjoint square root $s$ of $g^{*} g$. Letting $u=g s^{-1}$, one finds

$$
u^{*} u=\left(g s^{-1}\right)^{*}\left(g s^{-1}\right)=s^{-1} g^{*} g s^{-1}=1
$$

as $g^{*} g=s^{2}$, and thus $u$ is orthogonal with respect to $*$ (which might be called unitary here). Moreover, it is usually possible to choose $s$ in a consistent fashion 
when $g$ varies, and the decomposition turns out to be continuous. Using the assignment $g \mapsto u$ to map $\mathrm{GL}_{n}$ onto $U_{n}$, one finds the connectedness of the latter.

An analog of the Cartan decomposition was used implicitly in BBC07] with

$$
g^{*}=g^{-i}
$$

for arbitrary elements $g$ and a fixed involution $i$ of a given group $G$ of finite Morley rank. This operation on elements of the (ambient) group behaves formally like an adjunction as one sees immediately that $\left(g g^{\prime}\right)^{*}=g^{* *} g^{*}$ for any elements $g$ and $g^{\prime}$ and that $1^{* *}=1$. Using this notation, one notices also that

$$
g^{*} g=i g^{-1} i g=i i^{g}
$$

i.e., $g^{*} g$ is nothing else than a strongly real element $i i^{g}$, inverted by the involution $i$ and its conjugate $i^{g}$. We further remark that with our definition the unitary group " $U(*)$ " corresponds naturally to $C(i)$ and that the set of selfadjoint automorphisms corresponds to the set of strongly real elements inverted by $i$. In our abstract context, the only technical point consists thus in finding a well defined, and definable, square root function corresponding to the extraction of square roots of $i i^{g}=g^{*} g$.

We prove the following theorem using this decomposition.

Theorem 31 Let $G$ be a connected group of finite Morley rank in which commuting is an equivalence relation on the set of involutions, and with no nontrivial normal 2-unipotent subgroup. Then $C(i)$ is connected for any involution $i$ of $G$.

Before passing to the proof of Theorem 31, it is worth commenting on the assumption of the absence of a normal 2-unipotent subgroup. In fact, the conclusion of Theorem 31 may fail if one drops this assumption, as the following example shows. If $K$ is an algebraically closed field of characteristic 2 , then in the connected solvable group of matrices

$$
\left\{\left(\begin{array}{cc}
t & a \\
0 & t^{4}
\end{array}\right): t \in K^{\times}, a \in K_{+}\right\}
$$

one sees that the centralizer of every involution is the cyclic extension of order 3 of the 2-unipotent subgroup (consisting of strictly upper triangular matrices). In particular centralizers of involutions are not connected in this group, even though commutation is an equivalence relation on the set of involutions.

Lemma 32 Let $G$ be a connected group of finite Morley rank in which commutation is an equivalence relation on the set of involutions, and assume there exists a noncentral involution $i$ of $G$. Then, either

(1) The definable subset $X$ of elements $g$ of $G$ such that $Z\left(C\left(i i^{g}\right)\right)$ contains no involutions is generic in $G$, or

(2) $i^{G}$ generates an infinite normal elementary abelian 2-group. 
Proof. Assuming we are not in case (1), then the definable subset $Y$ of elements $g$ such that $Z\left(C\left(i i^{g}\right)\right)$ contains an involution, that is the complement of $X$, is generic in $G$. Notice that $Y$ is a union of right cosets of $C(i)$.

For any $g$ in $Y, Z\left(C\left(i i^{g}\right)\right)$ contains a nontrivial Sylow 2-subgroup, normalized by $i$ and $i^{g}$. Hence each involution $i$ and $i^{g}$ has to commute with an involution in $Z\left(C\left(i i^{g}\right)\right)$, and the transitivity of the commutation of involutions implies that $i$ and $i^{g}$ commute. Now the transitivity of the commutation of involutions forces that any two involutions $i^{g_{1}}$ and $i^{g_{2}}$, where $g_{1}$ and $g_{2} \in Y$, have to commute also. In particular $i^{Y}$ is a set of commuting involutions. As $Y$ is generic in $G$, $i^{Y}$ is generic in $i^{G}$.

Now $i^{G}$ is infinite as $C(i)<G$ by assumption, and $i^{G}$ has Morley degree one as it is in definable bijection with $G / C(i)$. As $i^{Y}$ is generic in $i^{G}$, it follows that any two $G$-conjugates of $i^{Y}$ intersect on a subset generic in $i^{G}$, and thus in particular any two such conjugates, consisting of pairwise commuting involutions, commute by the transitivity of the commutation on the set of involutions. As $G$ acts transitively by conjugation on $i^{G}$, it follows that $i^{G}$ consists of pairwise commuting involutions. But $i^{G}$ is a normal subset of $G$. Hence we conclude that $i^{G}$ generates an infinite elementary abelian 2-group, normal in $G$. We are thus necessarily in case (2).

It remains just to show that cases (1) and (2) are mutually exclusive. But in case (2), one has that $i i^{g}$ is an involution, necessarily in $Z\left(C\left(i i^{g}\right)\right)$, for any $g$ in $G \backslash C(i)$. Hence $X \subseteq C(i)$, and $X$ is in particular not generic in $G$

Proof of Theorem [31. If $i$ belongs to the center of $G$, then $C(i)=G$ is connected. Assume now $C(i)<G$. By Lemma 32, the definable subset $X$ of elements $g$ of $G$ such that $Z\left(C\left(i i^{g}\right)\right)$ contains no involutions is generic. Notice that $X$ is a union of right cosets of $C(i)$. We can define the Borovik-Cartan function associated to the operation

$$
g^{*}=g^{-i} .
$$

For $g$ in $X$, there exists a unique square root $s$ of $i i^{g}=g^{*} g$ in $Z\left(C\left(i i^{g}\right)\right)$, and the function

$$
\psi: g \mapsto g s^{-1}
$$

from $X$ to $G$ is well defined and definable. We also note that the square root $s$ is necessarily in the subgroup of $Z\left(C\left(i i^{g}\right)\right)$ of elements inverted by $i$, as this subgroup has no involutions also, and thus $s^{*}=s$ and $\left(s^{-1}\right)^{*}=s^{-1}$. We then get

$$
\psi(g)^{*} \psi(g)=\left(g s^{-1}\right)^{*} g s^{-1}=s^{-1} g^{*} g s^{-1}=1
$$

as $g^{*} g=s^{2}$. In other words, the function $\psi$ takes all its values in $C(i)$.

Furthermore, for any $c$ in $C(i)$ one has

$$
(c g)^{*}(c g)=g^{*} c^{*} c g=g^{*} g,
$$


so that the square roots $s_{c g}$ and $s_{g}$ of $(c g)^{*}$ and $g^{*}$ are the same. This shows the following covariant property:

$$
\psi(c g)=(c g) s_{c g}^{-1}=(c g) s_{g}^{-1}=c\left(g s_{g}^{-1}\right)=c \psi(g) .
$$

In particular, the fibers of $\psi$ are of constant rank, say $f$, and any subset of $C(i)$ of rank $r$ lifts to a subset of $X$ of rank $r+f$. If $C(i)$ were not connected, then it would have two disjoint generic subsets of full rank, which would necessarily lift to disjoint generic subsets of $X$, contradicting the connectedness of $G$. Hence $C(i)$ is connected.

In connection of Theorem 31 , it is worth mentioning the so-called $Z^{*}$-theorem (correcting also a slightly inacurate statement in [BBC07]). We say that an involution $k$ of a group $G$ of finite Morley rank is isolated whenever

$$
\left|k^{G} \cap S\right|=1
$$

for some (any) Sylow 2-subgroup $S$ of $G$.

Fact 33 ( $Z^{*}$-theorem [BBC07, Theorem 6]) Let $G$ be a connected group of finite Morley rank. If some involution $k$ of $G$ is isolated, then $C(k)$ is connected.

We stress the fact that one only has an implication as in Fact 33 , and not an alternative "either $k$ is not isolated or $C(k)$ is connected" as suggested by the statement used in [BBC07, Theorem 6]. In fact there are connected groups of finite Morley rank with non-isolated involutions $k$ and with $C(k)$ connected, as the following examples show. In $\mathrm{SL}_{2}$ over some algebraically closed field of characteristic 2, centralizers of involutions are connected and all involutions of the elementary abelian 2-subgroups are conjugate at the same time. Another example is provided by $\mathrm{GL}_{2}$ over an algebraically closed field of characteristic different from 2 , where the centralizer of the involution

$$
\left(\begin{array}{ll}
1 & 0 \\
0 & -1
\end{array}\right)
$$

is the definable subgroup of diagonal matrices (a connected torus), and this involution is conjugate to the other non-central involution

$$
\left(\begin{array}{ll}
-1 & 0 \\
0 & 1
\end{array}\right)
$$

To conclude, we mention the following special case of Theorem 31, which will be used notably in our reworking of [BCJ07, Case I] in [DJ07b].

Corollary 34 Let $G$ be a connected group of finite Morley rank such that $U_{2}(F(G))=$ 1. 
(1) If $G$ contains a strongly embedded subgroup in which an involution is central, then the centralizers of involutions of $G$ are connected.

(2) In particular, if $G$ has odd type, Prüfer 2-rank 1, and connected Sylow 2 -subgroups, then the centralizers of involutions of $G$ are connected.

Proof. (1). Let $M$ denote the strongly embedded subgroup of $G$. By assumption, $M=C(i)$ for some of its involutions, and as all involution of $M$ are $M$-conjugate ([BN94, Theorem 10.19]) this holds for any involution of $M$. We also note that the set of involutions of $M$ form an elementary abelian 2-group (central in $M$ ). By strong embedding of $M$, we also see that any two distinct conjugates of the elementary abelian 2-subgroup of $M$ have a trivial intersection. Hence Theorem 31 gives the desired result.

(2). By assumption, a Sylow 2-subgroup $S$ of $G$ has the isomorphism type

$$
S \simeq \mathbb{Z}_{2^{\infty}} .
$$

Let $i$ denote the unique involution of $G$, and $M=C(i)$. As $i$ is the unique involution of $M, M$ is strongly embedded in $G$. Hence we are in a special case of case (1).

We note that Corollary 34 (2) follows also more generally from the recent proof that $C(i) / C^{\circ}(i)$ has exponent at most 2 for any involution $i$ in a connected group of finite Morley rank of odd type [Del08a]. Actually, this result implies, more generally, that centralizers of involutions are connected in any connected groups of finite Morley rank of odd type with connected Sylow 2-subgroups.

\section{References}

[ABC97] T. Altınel, A. Borovik, and G. Cherlin. Groups of mixed type. J. Algebra, 192(2):524-571, 1997.

[ABC99] T. Altınel, A. Borovik, and G. Cherlin. On groups of finite Morley rank with weakly embedded subgroups. J. Algebra, 211(2):409-456, 1999.

[ABC08] T. Altınel, A. V. Borovik, and G. Cherlin. Simple groups of finite Morley rank, volume 145 of Mathematical Surveys and Monographs. American Mathematical Society, Providence, RI, 2008.

[AC99] T. Altinel and G. Cherlin. On central extensions of algebraic groups. J. Symbolic Logic, 64(1):68-74, 1999.

[Alt96] T. Altinel. Groups of finite Morley rank with strongly embedded subgroups. J. Algebra, 180(3):778-807, 1996. 
[BBC07] A. Borovik, J. Burdges, and G. Cherlin. Involutions in groups of finite Morley rank of degenerate type. Selecta Math. (N.S.), 13(1):1-22, 2007.

[BC07] J. Burdges and G. Cherlin. Semisimple torsion in groups of finite Morley rank. Preprint: arXiv:0801.3953, 2007.

[BC08] A. Borovik and G. Cherlin. Permutation groups of finite Morley rank. In Z. Chatzidakis, H.D. Macpherson, A. Pillay, and A.J. Wilkie, editors, Model Theory with applications to algebra and analysis, I and II. Cambridge University Press, Cambridge, 2008.

[BCJ07] J. Burdges, G. Cherlin, and E. Jaligot. Minimal connected simple groups of finite Morley rank with strongly embedded subgroups. $J$. Algebra, 314(2):581-612, 2007.

[BN94] A. Borovik and A. Nesin. Groups of finite Morley rank. The Clarendon Press Oxford University Press, New York, 1994. Oxford Science Publications.

[BP90] A. V. Borovik and B. P. Poizat. Tores et p-groupes. J. Symbolic Logic, 55(2):478-491, 1990.

[CJ04] G. Cherlin and E. Jaligot. Tame minimal simple groups of finite Morley rank. J. Algebra, 276(1):13-79, 2004.

[Del07a] A. Deloro. Groupes simples connexes minimaux algébriques de type impair. J. Algebra, 317(2):877-923, 2007.

[Del07b] A. Deloro. Groupes simples connexes minimaux de type impair. Thèse de doctorat, Université de Paris 7, 2007.

[Del08a] A. Deloro. Bagatelle in finite Morley rank. Submitted, 2008.

[Del08b] A. Deloro. Groupes simples connexes minimaux non-algébriques de type impair. J. Algebra, 319(4):1636-1684, 2008.

[DJ07a] A. Deloro and E. Jaligot. Groups of finite Morley rank with solvable local subgroups. Submitted: arXiv:0802.1394, 2007.

[DJ07b] A. Deloro and E. Jaligot. Groups of finite Morley rank with solvable local subgroups and of odd type. In preparation, 2007.

[FJ05] O. Frécon and E. Jaligot. The existence of Carter subgroups in groups of finite Morley rank. J. Group Theory, 8(5):623-644, 2005.

[FJ08] O. Frécon and E. Jaligot. Conjugacy in groups of finite Morley rank. In Z. Chatzidakis, H.D. Macpherson, A. Pillay, and A.J. Wilkie, editors, Model Theory with applications to algebra and analysis, I and II. Cambridge University Press, Cambridge, 2008. 
[Jal99] E. Jaligot. Groupes de type mixte. J. Algebra, 212(2):753-768, 1999.

[Jal00] E. Jaligot. FT-groupes. Prépublication de l'Institut Girard Desargues 33, CNRS UPRESA 5028, Lyon, Janvier 2000, http://math.univ-lyon1.fr/ jaligot/publ.html, 2000.

[Jal01a] E. Jaligot. Full Frobenius groups of finite Morley rank and the FeitThompson theorem. Bull. Symbolic Logic, 7(3):315-328, 2001.

[Jal01b] E. Jaligot. Groupes de rang de Morley fini de type pair avec un sousgroupe faiblement inclus. J. Algebra, 240(2):413-444, 2001.

[Jal08a] E. Jaligot. Cosets, genericity, and the weyl group. Submitted: arXiv:0801.2243, 2008.

[Jal08b] E. Jaligot. Simple groups of finite Morley rank. In Logic Colloquium 2005, volume 28 of Lect. Notes Log., pages 57-67. Assoc. Symbol. Logic, Urbana, IL, 2008.

[Nes90a] A. Nesin. On sharply $n$-transitive superstable groups. J. Pure Appl. Algebra, 69(1):73-88, 1990.

[Nes90b] A. Nesin. On solvable groups of finite Morley rank. Trans. Amer. Math. Soc., 321(2):659-690, 1990.

[Tho68] J. G. Thompson. Nonsolvable finite groups all of whose local subgroups are solvable. Bull. Amer. Math. Soc., 74:383-437, 1968.

[Tho70] J. G. Thompson. Nonsolvable finite groups all of whose local subgroups are solvable. II. Pacific J. Math., 33:451-536, 1970.

[Tho71] J. G. Thompson. Nonsolvable finite groups all of whose local subgroups are solvable. III. Pacific J. Math., 39:483-534, 1971.

[Tho73] J. G. Thompson. Nonsolvable finite groups all of whose local subgroups are solvable. IV, V, VI. Pacific J. Math., 48:511-592, 50 (1974) 215-297, 51 (1974) 573-630, 1973. 